\title{
Fourier-Based Forward and Back-Projectors in Iterative Fan-Beam Tomographic Image Reconstruction
}

\author{
Yingying Zhang-O'Connor*, Student Member, IEEE, and Jeffrey A. Fessler, Fellow, IEEE
}

\begin{abstract}
Fourier-based forward and back-projection methods can reduce computation in iterative tomographic image reconstruction. Recently, an optimized nonuniform fast Fourier transform (NUFFT) approach was shown to yield accurate parallel-beam projections. In this paper, we extend the NUFFT approach to describe an $O\left(N^{2} \log N\right)$ projector/backprojector pair for fan-beam transmission tomography. Simulations and experiments with real CT data show that fan-beam Fourier-based forward and back-projection methods can reduce computation for iterative reconstruction while still providing accuracy comparable to their $O\left(N^{3}\right)$ space-based counterparts.
\end{abstract}

Index Terms-Fan-beam tomography, iterative tomographic image reconstruction, min-max interpolation, nonuniform FFT.

\section{INTRODUCTION}

$\mathbf{T}$ HE CLASSICAL approach to reconstructing fan-beam tomography images is filtered back-projection (FBP). Statistical image reconstruction methods are based on models for measurement statistics and physics, and offer advantages such as the potential for improved image quality and reduced dose. A drawback of statistical image reconstruction methods is the longer computation time of iterative algorithms.

Most iterative algorithms require one forward projection and one back-projection each iteration, and these steps are the computational bottleneck. Using the Fourier-slice theorem for parallel-beam tomography, Matej et al. evaluated forward and back projectors [1]-[4] that used a nonuniform fast Fourier transform (NUFFT) with a min-max optimized Kaiser-Bessel (KB) interpolation kernel [5]. Their results showed low interpolation error and good computation efficiency. This paper extends the NUFFT approach to the fan-beam geometry that is used widely in X-ray computed tomography (CT) systems [6].

There are two complications in extending Fourier-based projectors to a fan-beam geometry. Firstly, in the fan-beam case there is no suitable Fourier slice theorem. (A recent extension is suitable for analytical reconstruction [7], but it is not evident

Manuscript received September 23, 2005; revised January 17, 2006. This work was supported in part by GE Medical Systems and in part by the National Institutes of Health (NIH) under Grant CA87634 and Grant EB00218. Asterisk indicates corresponding author.

*Y. Zhang-O'Connor is with the Department of Electrical Engineering and Computer Science, University of Michigan, Ann Arbor, MI 48109-2122 USA (e-mail: yzz@umich.edu).

J. A. Fessler is with the Department of Electrical Engineering and Computer Science, University of Michigan, Ann Arbor, MI 48109-2122 USA (e-mail: fessler@umich.edu).

Digital Object Identifier 10.1109/TMI.2006.872139 how to use it for forward projection.) Therefore, we use the usual Fourier slice theorem and interpolate into the fan-beam coordinates. To ensure good accuracy and computation efficiency, we use a min-max optimized KB NUFFT approach for the radial "interpolation." The second complication is that Fouriermethods are efficient only for shift-invariant detector response models. In emission tomography with converging (fan-beam) collimators, the detector response is highly shift variant, and it is unlikely that Fourier-based methods can be suitable. We focus here on transmission tomography for the fan-beam geometries of typical clinical X-ray CT scanners, where the variation in detector response over the field of view is often fairly modest. We approximate the detector response by the effective width at the center of the field of view, and investigate the effect of this approximation.

We focus on iterative reconstruction but we note for completeness that NUFFT-based direct Fourier image reconstruction methods have also been investigated [8], [9].

The paper is organized as follows. Section II describes the algorithm for the fan-beam case. Section III gives simulation and real data reconstruction results, including an accuracy comparison of NUFFT-based, space-based and analytically computed forward and back-projections, both as stand-alone modules and within iterations.

\section{AlgORITHM}

This section describes the steps for fan-beam NUFFT-based forward projection, as summarized in Fig. 2.

\section{A. Fourier Slice Theorem}

The Fourier slice theorem [10] is the foundation of Fourier-based forward projection. Let $g(x, y)$ denote the two-dimensional (2-D) image and $G_{\theta}(\rho)$ denote its 2-D FT in polar coordinates

$$
G_{\theta}(\rho) \triangleq \iint g(x, y) \mathrm{e}^{-\imath 2 \pi \rho(x \cos \theta+y \sin \theta)} \mathrm{d} x \mathrm{~d} y .
$$

The ideal line-integral projection of $g(x, y)$ at angle $\theta$ (taken counter-clockwise from the $y$ axis) as a function of the radial distance $r$ from the origin is given by

$$
p_{\theta}(r)=\int g(r \cos \theta-\ell \sin \theta, r \sin \theta+\ell \cos \theta) \mathrm{d} \ell .
$$




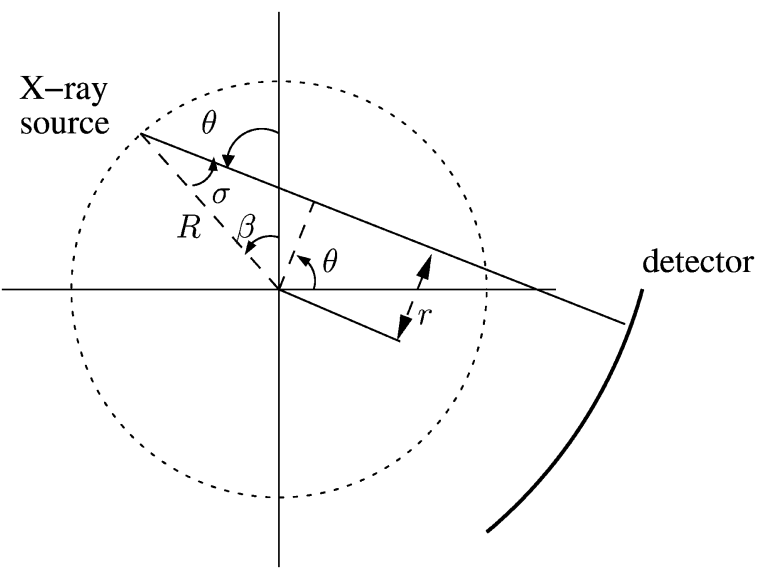

Fig. 1. Angular coordinates in fan-beam geometry.

For our purposes, the most convenient form of the Fourier slice theorem expresses each projection as a one-dimensional (1-D) inverse Fourier transform of $G_{\theta}(\rho)$ as follows:

$$
p_{\theta}(r)=\int G_{\theta}(\rho) \mathrm{e}^{\imath 2 \pi \rho r} \mathrm{~d} \rho .
$$

Fourier-based projectors use discretized versions of (1) and (3), whereas space-based projectors discretize (2).

\section{B. Fan-Beam Tomography}

As illustrated in Fig. 1, fan-beam rays are indexed by angular coordinates $(\beta, \sigma)$, where $\beta$ is the angle of the source relative to the $y$ axis, and $\sigma$ is the angle of the ray relative to the source. Fan-beam FBP methods exist only for special detector configurations [11]. Here, we accommodate any set of samples $\sigma_{m}$, $m=1, \ldots, N_{\sigma}$. We assume that $\beta$ is sampled uniformly, i.e., $\beta=2 \pi\left(k / N_{\beta}\right), k=0, \ldots, N_{\beta}-1$, although this could be relaxed.

To develop a Fourier-based projector for fan-beam geometries, we use the well-known relation between parallel-beam and fan-beam coordinates [12]

$$
\begin{aligned}
& r=R \sin \sigma \\
& \theta=\beta+\sigma
\end{aligned}
$$

where $R$ is the source to rotation center distance, illustrated in Fig. 1. For typical angular samples $\left\{\sigma_{m}\right\}$, the corresponding radial samples $r_{m} \triangleq R \sin \sigma_{m}$ are spaced nonuniformly. And when $\beta$ is sampled uniformly, for a given $\sigma_{m}$ (or equivalently a given $r_{m}$ ), the corresponding values of $\theta$ are also equally spaced but shifted by $\sigma_{m}$.

\section{NUFFT-Based Fan-Beam Forward Projector}

Fig. 2 shows the four major steps of the proposed fan-beam NUFFT-based forward projector. These four steps are summarized next.

1) Two-Dimensional NUFFT : Step 1 in Fig. 2 uses a 2-D NUFFT to evaluate a discretized version of (1). The input is $N_{1} \times N_{2}$ samples of the image $g(x, y)$. The output is polar coordinate samples of $G_{\theta}(\rho)$ that are equally-spaced along $\rho$ at locations $\left\{n \triangle_{\rho}\right\}$, for $n=-N_{\rho} / 2, \ldots, N_{\rho} / 2-1$, where $\triangle_{\rho}$ is the sample spacing in $\rho . N_{\rho}$ is chosen based on the choice of $\triangle_{\rho}$ and the extent of the spectral samples. In light of (5), we use $\theta$ samples that match the $\beta$ samples, i.e. $\theta_{k}=2 \pi\left(k / N_{\beta}\right)$.

Step 1 is identical to the parallel-beam case that is detailed in [1]-[5]. It involves the following operations.

a) Multiply the 2-D image samples by a scaling function that precompensates for imperfections in the frequency-domain interpolator. We use separable KB scaling functions for simplicity [5].

b) Calculate a $K_{1} \times K_{2}$ point (oversampled) 2-D FFT of the scaled discrete image. Typically $K_{1}=2 N_{1}$ and $K_{2}=$ $2 \mathrm{~N}_{2}$.

c) Interpolate onto the desired, nonuniformly-spaced frequency locations from the $J \times J$ nearest neighbors, using the $\mathrm{KB}$ interpolator that minimizes the worst-case approximation error [13], [14].

d) Multiply by the spectrum of the image-domain basis function (typically square pixels).

By choosing sufficient oversampling and sufficiently large $J \times J$ neighborhood, very high accuracy is achieved [4], [5].

2) Approximating Detector Response: In parallel-beam tomography, one can model shift-invariant detector blur in the frequency domain by multiplying the image spectrum $G_{\theta}(\rho)$ with the frequency response of the detector blur [4]. In fan-beam tomography, detector blur effects depend on the distances between each image pixel and the detector elements, and hence cannot be modeled exactly in the frequency domain. For example, in the CT system described in Section III, the effective detector width varies from $0.22 \mathrm{~mm}$ to $0.64 \mathrm{~mm}$ over a $40 \mathrm{~cm}$ field of view. For simplicity, we approximate the depth-dependent detector response by the effective beam width at the rotation center, calculated by multiplying the actual detector width by the ratio of the source-to-isocenter distance over the source-to-detector distance.

Simulations in Section III evaluate the effects of this shiftinvariant approximation.

The next two steps in Fig. 2 also differ between the parallelbeam and fan-beam geometries.

3) One-Dimensional Nonuniform IFFTs: Step 3 in Fig. 2 evaluates a discretized version of (3). In the parallel-beam case, a simple 1-D inverse FFT along $r$ for each $\theta_{k}$ will suffice [4]. For the fan-beam case, the desired radial samples $r_{m}$ are spaced unequally, per [4]. We discretize (3) as follows for $m=1, \ldots, M$ :

$$
p_{\theta_{k}}\left(r_{m}\right) \approx \sum_{n=-\frac{N_{\rho}}{2}}^{\frac{N_{\rho}}{2}} G_{\theta_{k}}\left(n \triangle_{\rho}\right) \triangle_{\rho} \mathrm{e}^{\imath 2 \pi \triangle_{\rho} n r_{m}}
$$

where we choose $\triangle_{\rho} \leq\left(1 / 2 r_{\max }\right)$ to avoid aliasing.

Since the $r_{m}$ values are spaced unequally, whereas the $\rho_{n}=$ $n \triangle_{\rho}$ values are spaced equally, we evaluate (6) for each $\theta_{k}$ using a 1-D NUFFT with "frequency" locations $-2 \pi \triangle_{\rho} r_{m}$ [5].

4) One-Dimensional Shifts Using FFT-Based Interpolations: After step 3 in Fig. 2, we have projection data that is nonuniformly spaced in $r$ (corresponding to each $\sigma_{m}$ ), and uniformlyspaced in $\theta$. For each $m=1, \ldots, N_{\sigma}$, a 1-D shift [by $\sigma_{m}$, see 


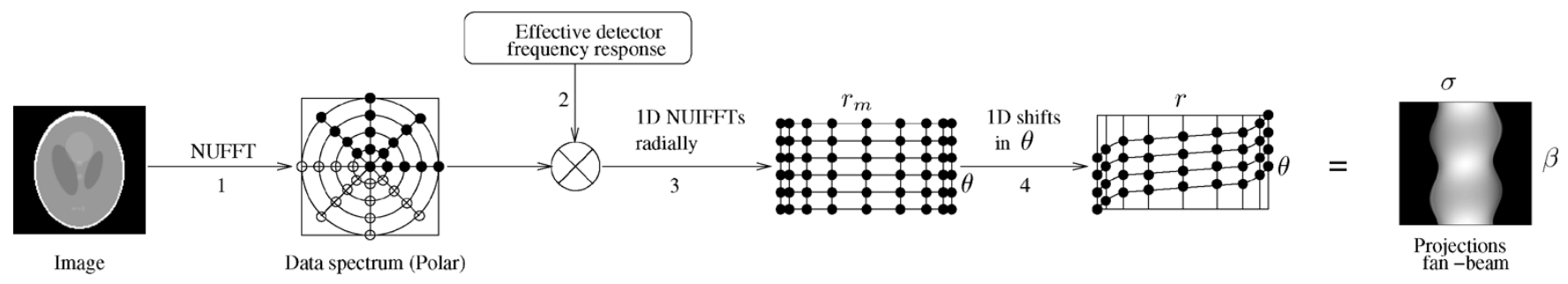

Fig. 2. Basic steps of NUFFT forward projection in fan-beam CT. 1) Two-dimensional NUFFT of image to obtain polar spectrum samples. 2) Multiply radially by the frequency response of the effective detector blur. 3) One-dimensional NUFFTs along radial direction $r$ for each $\theta$. 4) One-dimensional shifts in $\theta$ using interpolation with periodic end condition.

TABLE I

FLOATING-POINT OPERATIONS FOR NUFFT-BASED AND SPACE-BASED ForWARD PROJECTORS. ASSUME $N \times N$ IMAGE AND $N_{\rho} \approx N_{\sigma}$

\begin{tabular}{c|c|c||c}
\hline \multicolumn{3}{|c||}{ NUFFT-based } & space-based \\
steps & FFT & interpolation & (line integral) \\
\hline 2D NUFFT & $2 N^{2} \log N$ & $J^{2} N_{\sigma} N_{\beta} / 4$ & \\
1D NUFFTs & $N_{\beta} N_{\sigma} \log N_{\sigma}$ & $J_{1} N_{\sigma} N_{\beta}$ & $O$ \\
\cline { 2 - 3 } 1D shifts & \multicolumn{2}{|c|}{$\left.2 N_{\beta} \log N_{\beta} N_{\sigma} N\right)$} \\
\hline
\end{tabular}

(5)] in the $\theta$ direction is needed. Since projections are $2 \pi$ periodic in $\theta$, Dirichlet-like "periodic sinc" interpolation is a natural choice to "fractionally shift" the resulting projection data in the previous step into the desired $\theta$ locations. We use 1-D FFTs for this final step [15].

5) Symmetry Properties: Because the input image, $g(x, y)$ is real in CT, its Fourier transform, $G_{\theta}(\rho)$ is Hermitian symmetric. Thus, only half of the Fourier samples must be calculated in the 2-D NUFFT (step 1) and the 1-D NUFFT (step 3). In particular, we implement (3) as follows:

$p_{\theta_{k}}\left(r_{m}\right) \approx G_{\theta}(0)+2 \cdot \operatorname{real}\left\{\sum_{n=1}^{\frac{N_{\rho}}{2}} G_{\theta_{k}}\left(n \triangle_{\rho}\right) \triangle_{\rho} \mathrm{e}^{\imath 2 \pi \triangle_{\rho} n r_{m}}\right\}$.

This approach reduces computation and also ensures that the projections are entirely real valued. There may still be small negative values even if $g$ is nonnegative. For some iterative algorithms these may need be set to zero.

For further savings, we exploit the Radon symmetry property: $p_{\theta}(-r)=p_{\theta \pm \pi}(r)$. So we only need to compute the polar frequency samples for $\theta \in[0, \pi)$ instead of $[0,2 \pi)$ in step 1 shown in Fig. 2.

6) Backprojection: Iterative algorithms require repeated forward and back-projections, where the back-projector is the adjoint operator of the forward projector. We implement the backprojector by "reversing" (not inverting!) the linear steps above. This approach provides an exact adjoint, so the forward and back projectors are matched perfectly.

\section{Theoretical Analysis of Operation Flops}

Table I summarizes the dominant operation counts for the NUFFT-based forward projector. The expressions are for a $N \times$ $N$ image and a $N_{\sigma} \times N_{\beta}$ sinogram. We use a $J \times J$ neighborhood of 2-D DFT samples for interpolation in the 2-D NUFFT, and
$J_{1}$ neighbors for the 1-D NUFFT. The 2-D NUFFT computes $N_{\rho} \times N_{\beta} / 4$ polar frequency samples.

For comparison, a line-length space-based forward projector requires $O\left(N_{\beta} N_{\sigma} N\right)$ operations, where the proportionality constant can be large when the intersection lengths are computed on the fly (rather than precomputed) due to the very large image sizes in CT.

In the usual third-generation CT fan-beam geometry where $N_{\sigma} \approx 2 N$ and $N_{\beta} \approx 2 N$, the overall computation for the NUFFT-based forward projector is $O\left(N^{2} \log N\right)$, akin to previous hierarchical methods [16], whereas most space-based forward projectors require $O\left(N^{3}\right)$ operations.

\section{RESULTS}

We simulated a third-generation fan-beam X-ray CT system with sinogram size of approximately $1.7 \mathrm{~N}$ radial bins by $2 \mathrm{~N}$ views over $360^{\circ}$. The source to detector distance is about 949 $\mathrm{mm}$, and the rotation center to detector distance is about 408 $\mathrm{mm}$. Thus, $R=541 \mathrm{~mm}$. For example, when $N=512$, the corresponding sinogram size is 888 samples in $\sigma$, spaced by $\triangle_{\sigma} \approx$ $0.06^{\circ}$ and 984 source positions over $360^{\circ}$, so $\triangle_{\beta} \approx 0.37^{\circ}$. A quarter detector offset is also included to reduce aliasing. Except where noted below, we use $N_{\rho}=1.7 N$ and $\triangle_{\rho}=1 / \mathrm{FOV}$ in (7).

The Fourier-based method is implemented in Matlab (version 7.0.4) using double precision; the NUFFT spectral interpolator is an ANSI C MEX routine. The space-based projector is an ANSI C MEX routine using single precision. All projectors are evaluated on a Dell 670n computer with dual Intel Xeon 3.40 $\mathrm{GHz}$ CPU.

The space-based projector used here was designed originally for coordinate-wise algorithms, e.g., [17]. It was not optimized for ray-driven calculation of the line-integral model described in case 1 in Section III-A. Its primary role here is for accuracy comparisons.

\section{A. Forward and Back-Projector as Single Modules}

1) Forward Projector: We evaluated the NUFFT-based fanbeam forward and back-projectors using the Shepp-Logan digital phantom. The brain-size field of view is approximately 308 $\mathrm{mm}$, thus pixel size is about $0.6 \mathrm{~mm}$ for $N=512$. We investigated four different scenarios, involving three different analytical models. All cases used the analytical formula for the fan-beam projections of ellipses to provide a "gold standard" reference sinogram. 
TABLE II

SPACE-BASED AND NUFFT-BASEd FoRWARd PROJECTIONS OF A $512 \times 512$ IMAGE COMPARED TO EXACT ANALYTICAL PROJECTIONS FOR THREE DIFFERENT Analytical Models. Case 1: Analytical, Space-Based and NUFFT-Based Methods All Use Line InTEGRals. Case 2: Analytical Method

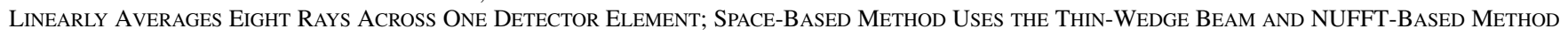
APPROXIMATES THE BEAM Width AT tHE Rotation CENTER. CASE 3a: ANALYTICAl METHOD NONLINEARLY AVERAGES EIGHT RAYS ACROSS ONE DETECTOR

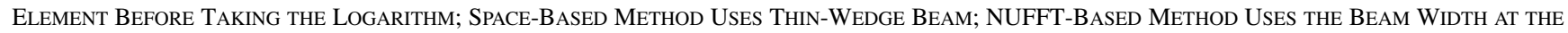
Rotation Center. Case 3b: As in CAse 3a Except NUFFT and Space-Based Methods Both Use Multiline Nonlinear Averaging

\begin{tabular}{c||c|c||c|c||c|c||c|c}
\hline \multicolumn{1}{c||}{ analytical models } & \multicolumn{2}{c||}{ Case 1 } & \multicolumn{2}{c||}{ Case 2 } & \multicolumn{2}{c||}{ Case 3a } & \multicolumn{2}{c}{ Case 3b } \\
\hline discretized models & $\begin{array}{c}\text { space } \\
\text { (line) }\end{array}$ & $\begin{array}{c}\text { NUFFT } \\
\text { (line) }\end{array}$ & $\begin{array}{c}\text { space } \\
\text { (beam) }\end{array}$ & $\begin{array}{c}\text { NUFFT } \\
\text { (center width) }\end{array}$ & $\begin{array}{c}\text { space } \\
\text { (beam) }\end{array}$ & $\begin{array}{c}\text { NUFFT } \\
\text { (center width) }\end{array}$ & $\begin{array}{c}\text { space } \\
\text { (multi-line) }\end{array}$ & $\begin{array}{c}\text { NUFFT } \\
\text { (multi-line) }\end{array}$ \\
\hline max \% error & 7.03 & 6.13 & 2.31 & 2.15 & 2.91 & 2.71 & 2.64 & 2.67 \\
$\ell_{1} \%$ error & 0.13 & 0.10 & 0.07 & 0.08 & 0.07 & 0.08 & 0.07 & 0.09 \\
$\%$ nrms error & 0.28 & 0.25 & 0.16 & 0.16 & 0.17 & 0.17 & 0.16 & 0.16 \\
\hline
\end{tabular}

Case 1-Line Integral Model: Here, we used the analytical formula for the fan-beam line-integral projections of an ellipse, sampled at the center of each detector element. For the NUFFT approach we set the detector frequency response to unity. For the space-based approach we set $a_{i j}$ to the usual length of intersection of the $i$ th ray with the $j$ th pixel. (This was implemented by setting the beam-width in Case 2 below to zero, rather than by developing an optimized, ray-driven line-integral algorithm.) This line-integral model is often used in evaluating forward projectors.

Case 2-Linear Averaging Model: The line-integral model is unrealistic since detectors have finite width and they average the incoming signal across that width. For Case 2, we generated the reference sinogram by linearly averaging 8 analytical rays sampled across each detector element. This also accounts for depth-dependent detector response. For the NUFFT method, we accounted for the finite beam width approximately by using the beam width at the center of the field of view. For the space-based approach, we computed $a_{i j}$ as the area of intersection between the $j$ th pixel and the thin-wedge connecting the point X-ray source with the $i$ th finite-width detector. That space-based model accounts for distance dependent beam-width but increases computation.

Case 3-Nonlinear Averaging Model: Due to the nonlinearity of Beer's law, the linear averaging model is imperfect. For Case 3, instead of linearly averaging the 8 analytically computed line integrals per detector element used in Case 2, we formed the reference sinogram by computing the negative logarithm of the average of the exponentials of the negatives of these ray values multiplied by $0.02 / \mathrm{mm}$. This nonlinear averaging introduces the "exponential edge gradient effect" that occurs in practice [18].

For this reference sinogram, we compared two different approaches to pixelized forward projectors. For Case 3a we used the same NUFFT and space-based forward projectors described under Case 2. Those projectors use linear averaging so increased errors are expected. For Case $3 b$ we used over-sampled versions of the line-integral models described under Case 1 and nonlinearly averaged the resulting line integrals over each detector element. These over-sampling approaches require much more computation than may be practical for routine use, but better match the nonlinearly averaged analytical sinogram. In this case, all of the methods account for distance-dependent beam width.
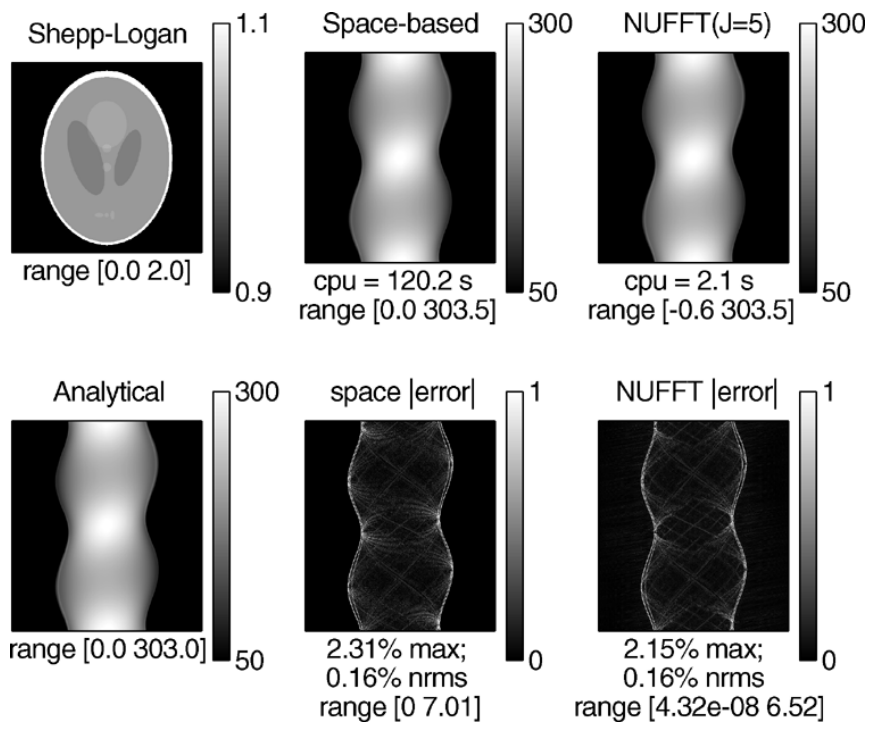

Fig. 3. Simulation results for forward projectors of image size $512 \times 512$ in Case 2: sinogram size of 888 bins by 984 views. The gray-scale ranges were chosen to show details. The exact ranges are shown below each image.

For all of the above scenarios, we computed the normalized maximum error, $\max _{j}\left(\left|x_{j}-y_{j}\right|\right) / \max _{j}\left(\left|x_{j}\right|\right)$, and the normalized $\ell_{p}$ error, $\left\|x_{j}-y_{j}\right\|_{p} /\left\|x_{j}\right\|_{p}$, specifically the normalized absolute error ( $\ell_{1}$ error) and the normalized root-mean-square (NRMS) error. For 1-D and 2-D NUFFT, we used FFT oversampling factor $K / N=2$ and the number of neighbor samples $J=5$. Table II summarizes the comparisons under these three different Cases. The space-based and NUFFT-based forward projectors perform similarly for all situations, despite the approximated detector response model.

We also performed the forward projections on various image sizes, $N=128,256,384,512$, and 1024. Fig. 3 shows the projections of an image of size $512^{2}$ in Case 2. The sinograms are visually indistinguishable. The accuracy of the NUFFT-based method is comparable with the space-based method, while the computation time (using Matlab cputime command) is about 57 times faster.

Table III further compares the computation times and accuracies for images of various sizes. To further demonstrate the computation efficiency, we also include the performance of the fast distance-driven (DD) projector proposed recently by De Man et al. [19], which is a $O\left(N^{3}\right)$ method, implemented as a C MEX 
TABLE III

COMPARISON OF SPACE-BASED, NUFFT-BASEd AND DD ForWARd PROJECTORS FOR VARIOUS IMAGE SIZES

\begin{tabular}{c||c|c|c|c||c|c|c||c|c|c}
\hline \multicolumn{1}{c||}{ Image size } & \multicolumn{3}{c||}{ CPU time (in seconds) } & \multicolumn{3}{c||}{ max \% error } & \multicolumn{3}{c}{$\%$ nrms error } \\
\cline { 2 - 9 } & \multicolumn{2}{c|}{ Space } & NUFFT & DD & $\begin{array}{c}\text { Space } \\
\text { (beam) }\end{array}$ & NUFFT & DD & $\begin{array}{c}\text { Space } \\
\text { (beam) }\end{array}$ & NUFFT & DD \\
\cline { 2 - 10 } & (line) & (beam) & & & & & & \\
\hline $128^{2}$ & 1.7 & 1.9 & 0.1 & 0.1 & 3.57 & 3.82 & 3.58 & 0.64 & 0.63 & 0.61 \\
$256^{2}$ & 13.5 & 15.2 & 0.5 & 0.7 & 3.05 & 3.76 & 3.05 & 0.31 & 0.31 & 0.30 \\
$384^{2}$ & 45.4 & 50.9 & 1.2 & 2.1 & 2.34 & 2.97 & 2.34 & 0.21 & 0.21 & 0.20 \\
$512^{2}$ & 106.9 & 120.2 & 2.1 & 5.0 & 2.31 & 2.15 & 2.31 & 0.16 & 0.16 & 0.15 \\
$1024^{2}$ & 852.5 & 956.3 & 8.6 & 39.2 & 1.53 & 1.58 & 1.53 & 0.08 & 0.08 & 0.07 \\
\hline
\end{tabular}

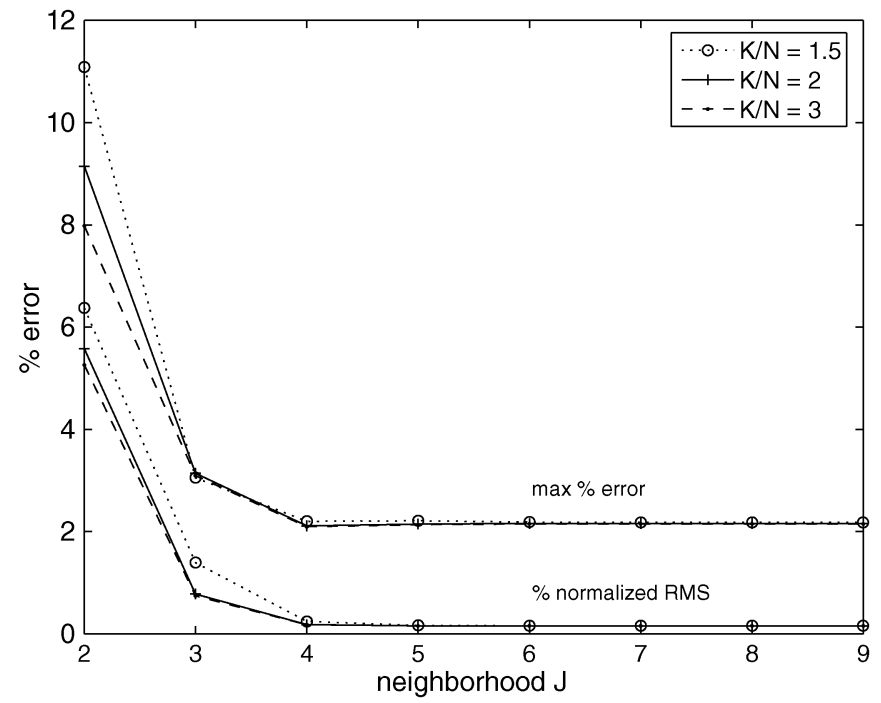

Fig. 4. Trade-off between NUFFT-based forward projector accuracy and oversampling factor $K / N$ and neighborhood $J \times J$ for $512^{2}$ image.

interface to $\mathrm{C}++$ code provided by those authors. The computation time of the NUFFT-based forward projector is comparable to the DD forward projector for small images and is about 2-4 times faster for larger images. The benefits of a $O\left(N^{2} \log N\right)$ method improves as $N$ increases, but in practice, the preferred method will depend on hardware considerations like pipelining.

Fig. 4 shows the accuracy as a function of the oversampling factor $K / N$ and the neighborhood size $J$. This plot suggests that $J=4$ would be adequate. However, this plot shows only the accuracy in the sinogram domain. In the image domain, some ringing artifacts appeared in the NUFFT image for $J=4$, suggesting that the sinogram domain does not adequately reveal high frequencies. Therefore, in all subsequent results, we chose $J=5$.

2) Back-Projector: To evaluate the backprojector, we processed the sinogram obtained from the analytical projections with the first two steps in the fan-beam FBP method. Since there is no easy way to calculate the exact back-projections analytically, here we applied only space-based and NUFFT-based methods to this processed sinogram.

In results not shown, the computation efficiencies and accuracies were very similar to those in Table III, as is expected because the back-projector is the exact adjoint of the forward projector. For a $512^{2}$ image, the back-projecton times for the space-based, NUFFT and DD methods were 245.2, 2.4 and 5.1 seconds, respectively.

\section{B. Forward and Back-Projectors Within Iterative Reconstruction}

Because even small approximation errors might accumulate after many iterations, it is necessary to evaluate the accuracy of the NUFFT-based projectors in iterative reconstruction methods.

1) Simulation Study: We used the analytical method mentioned under "Case 2" in Section III-A-1 to simulate a noiseless sinogram from a Shepp-Logan phantom. The sinogram size was 888 radial bins by 984 views over $360^{\circ}$. We ran 200 iterations of the conjugate gradient algorithm, initialized with $\mathbf{x}=\mathbf{0}$, for the following penalized weighted least-squares cost function with a quadratic roughness penalty (QPWLS-CG):

$$
\begin{aligned}
& \Phi(\mathbf{x})=\sum_{i=1}^{N_{d}} w_{i} \frac{1}{2}\left(y_{i}-[\mathbf{A} \mathbf{x}]_{i}\right)^{2}+\beta R(\mathbf{x}) \\
& R(\mathbf{x})=\sum_{k} \psi\left([\mathbf{C x}]_{k}\right)
\end{aligned}
$$

where $y_{i}$ is the negative $\log$ of the measured sinogram, $w_{i} \mathrm{~S}$ are statistical weighting factors, $\mathbf{A}$ is the system matrix, $\mathbf{C}$ is a differencing matrix and $\psi(t)$ is the potential function. Here, $\psi(t)=t^{2} / 2$, a quadratic penalty. For this simulation, we used $w_{i}=\exp \left(-\alpha[\mathbf{A x}]_{i}\right)$ where $\alpha$ is a scaling factor chosen to set the maximum value of $\alpha[\mathbf{A x}]_{i}$ to about 5. We chose $\beta=$ $2^{8}$ for this simulation. Evaluating the PSF using the approximations described in [20] shows that the FWHM is about 2.1 pixels, i.e., $1.3 \mathrm{~mm}$, for this value of $\beta$. We used the modified quadratic roughness penalty in [20] to obtain approximately uniform resolution.

We ran QPWLS-CG using the DD, space-based and NUFFTbased forward and back projectors respectively. Here, we used $N_{\rho}=1332$ in (7).

Fig. 5 shows that the reconstructed images are visually indistinguishable even with a 200-Hounsfield units (HU) window. Fig. 6 shows the profiles through the region of interest containing the small features in lower part of the phantom. The max percent difference between NUFFT-based and space-based and DD methods is less than $1.4 \%$ and NRMS is about $0.3 \%$. This difference is much smaller than the 3.7\% NRMS error of the PWLS estimates themselves (compared to the true object). The NUFFT method exhibits oscillations of about $1 \mathrm{HU}$ in this region. This could be reduced by increasing $N_{\rho}$ at the price of increased computation. The computation time is reduced by a factor of 80 for the NUFFT approach compared to 

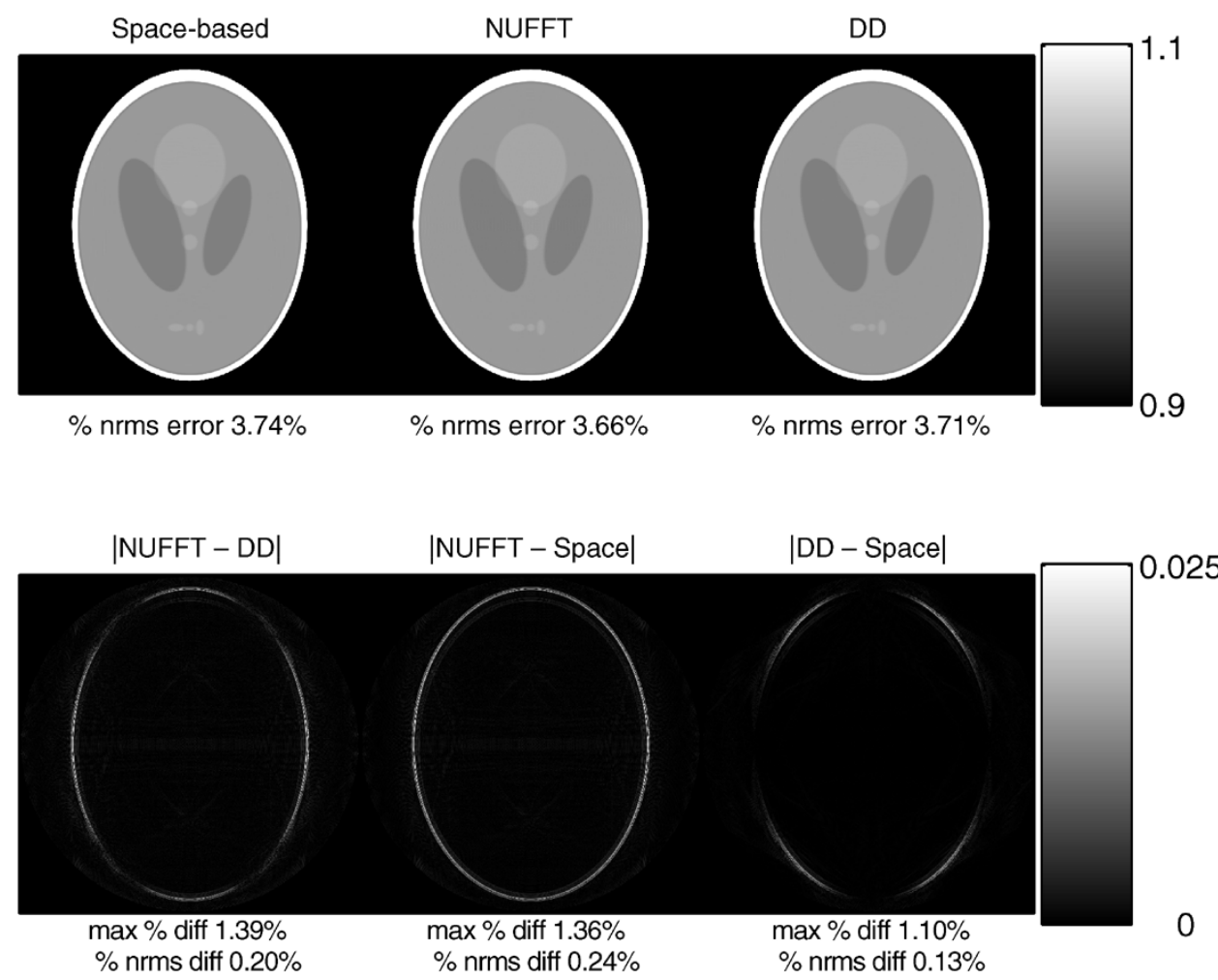

Fig. 5. QPWLS-CG reconstruction (200 iterations) for Shepp-Logan phantom with projectors from case 2: noiseless data, square pixels.

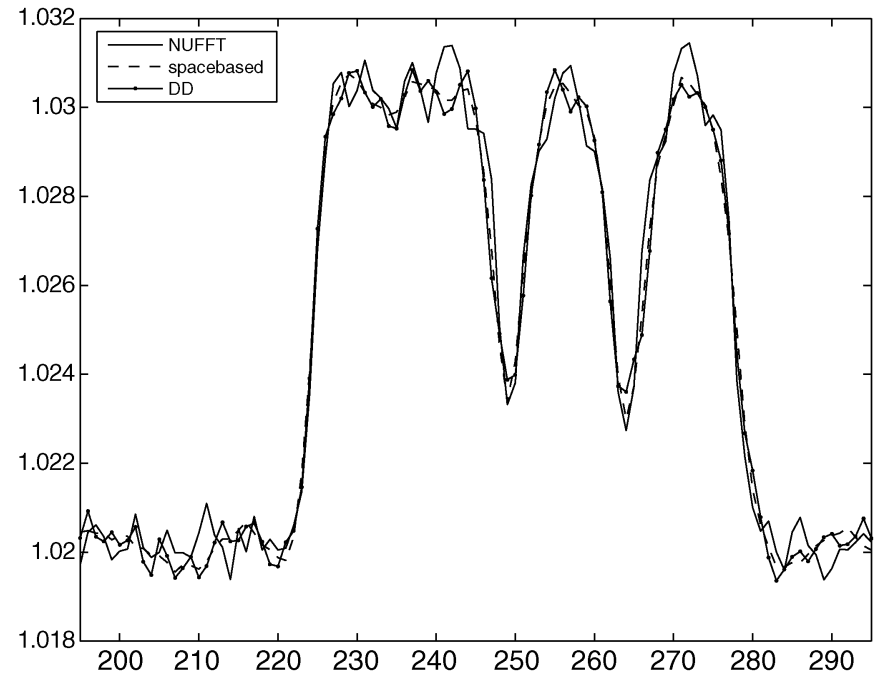

Fig. 6. Horizontal profiles through region of interest of the reconstructed images of DD, space-based and NUFFT-based methods at the 200th iteration.

the space-based method and a factor of 2 for the NUFFT approach compared to the DD method.

2) Real X-Ray CT Data: We further tested the NUFFT approach within iterative reconstruction using real (noisy) CT data. Transmission data from a human shoulder phantom were acquired on a GE Lightspeed scanner. The field of view is $500 \mathrm{~mm}$, thus, pixel size is about $1.0 \mathrm{~mm}$. Other parameters are as the same as those used in the previous noiseless data simulation. The initial image is the ramp-filtered FBP image with median filtering using a 3-by-3 neighborhood.
We ran 60 iterations of conjugate gradient algorithm for a PWLS cost function (PWLS-CG) [21]. We again use the expression in (8) and (9) except here we used an edge-preserving "hyperbola" penalty function

$$
\psi(t)=\frac{\delta^{2}}{3}\left(\sqrt{1+3\left(\frac{t}{\delta}\right)^{2}}-1\right) .
$$

The regularization parameters were $\delta=100 \mathrm{HU}$ and $\beta=2^{9}$ which gives FWHM 1.7 pixels, i.e., about $1.6 \mathrm{~mm}$. We also used the modified penalty described in [20]. We chose $w_{i}$ to correspond to the 2nd derivative of the transmission Poisson loglikelihood [22].

Fig. 7 shows the results of iterative reconstruction on real data with space-based and NUFFT-based projectors, respectively, using a standard display window width of $400 \mathrm{HU}$. The reconstructed images from the reconstruction methods with spacebased and NUFFT-based projectors are again visually indistinguishable, with the max difference less than $3.4 \%$ (178.3 HU) and NRMS around $1.0 \%$. The largest differences were at the edges of the field-of-view (FOV).

\section{DISCUSSION}

This paper has presented a NUFFT-based projection method for fan-beam tomography. This framework is an extension of parallel-beam NUFFT-based projectors. Our results show that the min-max NUFFT approach provides an accurate and efficient method for fan-beam forward and back-projection. Software is available online [23]. 

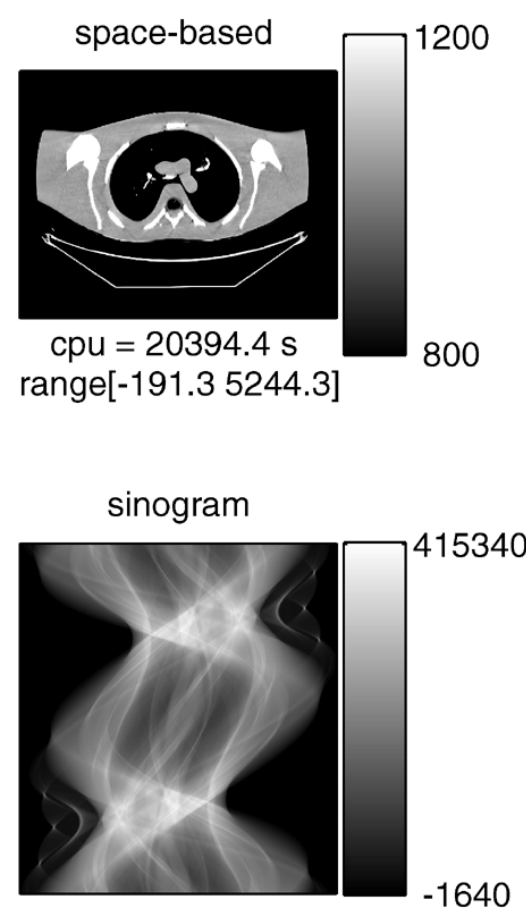
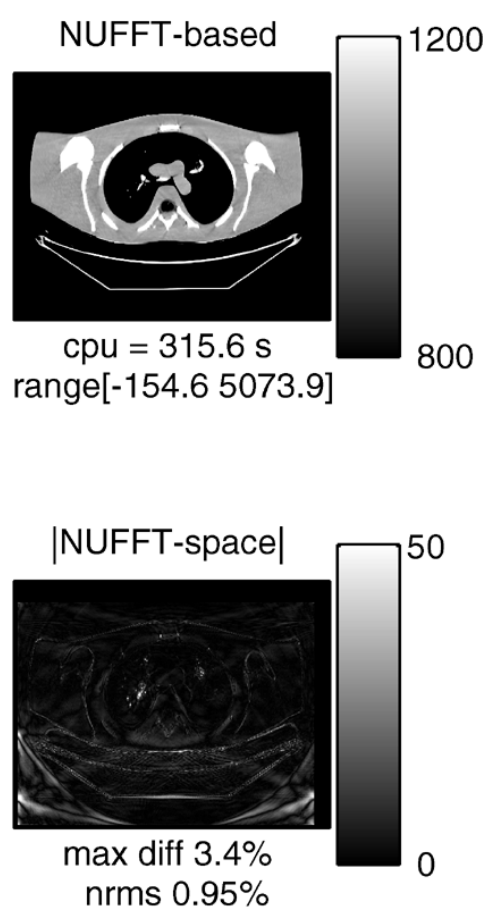

Fig. 7. PWLS-PCG reconstruction (60 iterations) for real X-ray CT fan-beam sinogram data with projectors from Case 2.

The NUFFT-based forward and back-projectors with min-max interpolation kernels are computationally efficient compared to space-based methods, and are reasonably accurate. As expected, the computation advantage increases with larger data size (Table III). The approximation error remains low even after many iterations. We have proposed this NUFFT approach for iterative fan-beam reconstruction. It is unclear how the NUFFT method could be used directly for fan-beam FBP, since that approach requires a weighted backprojection. However, one could first rebin from fan-beam to parallel-beam rays and then apply an NUFFT-type method.

In this paper we have used a NUFFT method that works generally for arbitrary sampling patterns. Further acceleration may be possible if one uses a specific 2-D NUFFT approach that is tailored to the polar sampling pattern required for the Radon transform, e.g., [24]. Many such methods have been proposed for direct Fourier reconstruction, e.g., [25]-[29]. Presumably some of these methods could be adapted to iterative reconstruction, for both parallel-beam and fan-beam geometries. The method also can be extended to the helical cone-beam geometry.

However, this method is poorly suited for "ordered-subsets" algorithms since it must compute an oversampled 2-D FFT even if only a few projecton views are needed. This property limits its application to algorithms where ordered-subsets are not needed. Existing $O\left(N^{2} \log N\right)$ methods also have this limitation [30].

\section{ACKNOWLEDGMENT}

The authors gratefully acknowledge J. Hsieh for providing the real data used in Section III-B2, and B. De Man and S. Basu for providing the code of the DD projector used in Section III-A.

\section{REFERENCES}

[1] J. A. Fessler and B. P. Sutton, "A min-max approach to the multidimensional nonuniform FFT: application to tomographic image reconstruction," in Proc. IEEE Int. Conf. Image Processing, 2001, vol. 1, pp. 706-709.

[2] J. A. Fessler, Iterative Tomographic Image Reconstruction Using Nonuniform Fast Fourier Transforms Tech. Rep., Comm. and Signal Process. Lab., Dept. EECS, Univ. Michigan, Ann Arbor, MI, 48 109-2122, Dec. 2001 [Online]. Available: http://www.eecs.umich. edu/ fessler, Technical report available from

[3] S. Matej, J. A. Fessler, and I. G. Kazantsev, Fourier-Based Forward and Back-Projectors for Iterative Image Reconstruction Univ. Pennsylvania, Philadelphia, Tech. Rep. MIPG303, May 2003.

[4] - "Iterative tomographic image reconstruction using Fourier-based forward and back-projectors," IEEE Trans. Med. Imag., vol. 23, no. 4, pp. 401-412, Apr. 2004.

[5] J. A. Fessler and B. P. Sutton, "Nonuniform fast Fourier transforms using min-max interpolation," IEEE Trans. Signal Process., vol. 51, no. 2, pp. 560-574, Feb. 2003.

[6] Y. Zhang and J. A. Fessler, "Fourier-based forward and back-projectors in iterative fan-beam tomographic image reconstruction," in Proc. IEEE Int. Symp. Biomedical Imaging, 2004, pp. 364-367.

[7] G.-H. Chen, S. Leng, and C. A. Mistretta, "A novel extension of the parallel-beam projection-slice theorem to divergent fan-beam and conebeam projections," Med. Phys., vol. 32, no. 3, pp. 654-665, Mar. 2005.

[8] S. De Francesco and A. M. Ferreira da Silva, "Efficient NUFFT-based direct Fourier algorithm for fan beam CT reconstruction," Proc. SPIE, vol. 5370, Medical Imaging 2004: Image Processing, pp. 666-677, 2004.

[9] K. Fourmont, "Non-equispaced fast Fourier transforms with applications to tomography," J. Fourier Anal. and Appl., vol. 9, no. 5, pp. 431-450, Sep. 2003.

[10] A. C. Kak and M. Slaney, Principles of Computerized Tomographic Imaging. Piscataway, NJ: IEEE Press, 1988.

[11] G. Besson, "CT fan-beam parametrizations leading to shift-invariant filtering," Inverse Prob., vol. 12, no. 6, pp. 815-833, Dec. 1996.

[12] H. Peng and H. Stark, "Direct Fourier reconstruction in fan-beam tomography," IEEE Trans. Med. Imag., vol. 6, no. 3, pp. 209-219, Sep. 1987.

[13] J. D. O'Sullivan, "A fast sinc function gridding algorithm for Fourier inversion in computer tomography," IEEE Trans. Med. Imag., vol. 4, no. 4, pp. 200-207, Dec. 1985. 
[14] J. I. Jackson, C. H. Meyer, D. G. Nishimura, and A. Macovski, "Selection of a convolution function for Fourier inversion using gridding," IEEE Trans. Med. Imag., vol. 10, no. 3, pp. 473-478, Sep. 1991.

[15] D. Fraser, "Interpolation by the FFT revisited. An experimental investigation," IEEE Trans. Acoust. Speech Signal Process., vol. 37, no. 5, pp. 665-676, May 1989.

[16] S. Basu and Y. Bresler, "Error analysis and performance optimization of fast hierarchical backprojection algorithms," IEEE Trans. Image Process., vol. 10, no. 7, pp. 1103-1117, Jul 2001.

[17] J. A. Fessler, E. P. Ficaro, N. H. Clinthorne, and K. Lange, "Grouped-coordinate ascent algorithms for penalized-likelihood transmission image reconstruction," IEEE Trans. Med. Imag., vol. 16, no. 2, pp. 166-175, Apr. 1997.

[18] P. M. Joseph and R. D. Spital, "The exponential edge-gradient effect in X-ray computed tomography," Phys. Med. Biol., vol. 26, no. 3, pp. 473-487, May 1981.

[19] B. De Man and S. Basu, "Distance-driven projection and backprojection in three dimensions," Phys. Med. Biol., vol. 49, no. 11, pp. 2463-2475, Jun 2004.

[20] J. A. Fessler and W. L. Rogers, "Spatial resolution properties of penalized-likelihood image reconstruction methods: space-invariant tomographs," IEEE Trans. Image Process., vol. 5, no. 9, pp. 1346-1358, Sep. 1996.

[21] J. A. Fessler and S. D. Booth, "Conjugate-gradient preconditioning methods for shift-variant PET image reconstruction," IEEE Trans. Image Process., vol. 8, no. 5, pp. 688-699, May 1999.
[22] K. Sauer and C. Bouman, "A local update strategy for iterative reconstruction from projections," IEEE Trans. Signal Process., vol. 41, no. 2, pp. 534-548, Feb. 1993.

[23] J. A. Fessler, Matlab Tomography Toolbox 2004 [Online]. Available: http://www.eecs.umich.edu/ fessler

[24] A. Averbuch and Y. Shkolnisky, "3D Fourier based discrete Radon transform," Appl. Computational Harmonic Anal., vol. 15, no. 1, pp. 33-69, Jul. 2003.

[25] W. Lawton, "A new polar Fourier transform for computer-aided tomography and spotlight synthetic aperture radar," IEEE Trans. Acoust. Speech Signal Process., vol. 36, no. 6, pp. 931-933, Jun. 1988.

[26] P. L. Bellon and S. Lanzavecchia, "Fast direct Fourier methods, based on one- and two-pass coordinate transformations, yield accurate reconstructions of X-ray CT clinical images," Phys. Med. Biol., vol. 42, no. 3, pp. 443-464, Mar. 1997.

[27] J. Waldén, "Analysis of the direct Fourier method for computer tomography," IEEE Trans. Med. Imag., vol. 19, no. 3, pp. 211-222, Mar. 2000.

[28] D. Potts and G. Steidl, "A new linogram algorithm for computerized tomography," IMA J. Numer. Anal., vol. 21, no. 3, pp. 769-782, Jul. 2001.

[29] G. Bal and P. Moireau, "Fast numerical inversion of the attenuated Radon transform with full and partial measurements," Inverse Prob., vol. 20, no. 4, pp. 1137-1164, Aug. 2004.

[30] S. Xiao, Y. Bresler, and D. Munson, " $O\left(N^{2} \log N\right)$ native fan-beam tomographic reconstruction," in Proc. IEEE Int. Symp. Biomedical Imaging, 2002, pp. 824-827. 